\title{
Berberine sensitizes rapamycin-mediated human hepatoma cell death in vitro
}

\author{
NA GUO $^{1 *}$, AILI YAN $^{1 *}$, XINGCHUN GAO $^{1 *}$, YANKE CHEN $^{2}$, XINYING HE $^{1}$, \\ ZHIFANG HU ${ }^{1}$, MAN MI ${ }^{1}, \mathrm{XU} \mathrm{TANG}^{3}$ and XINGCHUN GOU ${ }^{1}$ \\ ${ }^{1}$ Laboratory of Cell Biology and Translational Medicine, Institute of Basic Medical Science, X'ian Medical University, \\ Xi'an, Shaanxi 710021; ${ }^{2}$ Department of Cell Biology and Genetics, College of Medicine, Xi'an Jiaotong University, \\ Xi'an, Shaanxi 710032; ${ }^{3}$ Department of Pathology, Sichuan College of Traditional Chinese Medicine, \\ Mianyang, Sichuan 721000, P.R. China
}

Received September 18, 2013; Accepted April 24, 2014

DOI: $10.3892 / \mathrm{mmr} .2014 .2608$

\begin{abstract}
Rapamycin is clinically used as an immunosuppressant. Increasing evidence suggests that rapamycin has an important inhibitory role in the development and progression of different types of cancer and that it is a promising candidate for cancer chemotherapy. Berberine is an isoquinoline alkaloid isolated from medicinal plant species, which has been used in traditional Chinese medicine with no significant side effects. Recent research has demonstrated that berberine has anticancer activity against various types of cancer, mediated through the suppression of mammalian target of rapamycin (mTOR). The present study aimed to investigate the in vitro synergistic anticancer effect of combined treatment of rapamycin at various concentrations $(0,10,50,100$ and $200 \mathrm{nM})$ and berberine $(62.5 \mu \mathrm{M})$ in SMMC7721 and HepG2 hepatocellular carcinoma (HCC) cell lines, and the potential underlying molecular mechanism. The combined use of rapamycin and berberine was found to have a synergistic cytotoxic effect, with berberine observed to maintain the cyotoxic effect of rapamycin on HCC cells at a lower rapamycin concentration. Moreover, the cells treated with the combination of the two agents exhibited significantly decreased protein levels of phosphorylated (p)-p70S6 kinase 1 (Thr389), the downstream
\end{abstract}

Correspondence to: Professor Xingchun Gou, Laboratory of Cell Biology and Translational Medicine, Institute of Basic Medical Science, X'ian Medical University, 1 Xin Wang Road, Xi'an, Shaanxi 710021, P.R. China

E-mail: gouxingchun@189.cn

Xu Tang, Department of Pathology, Sichuan College of Traditional Chinese Medicine, 178 Changhong Avenue South, Mianyang, Sichuan 721000, P.R. China

E-mail: txxa7043@163.com

*Contributed equally

Key words: berberine, rapamycin, mammalian target of rapamycin, cluster of differentiation 147, human hepatoma cell effector of mTOR, compared with the cells treated with rapamycin or berberine alone. Furthermore, overexpression of cluster of differentiation (CD) 147, a transmembrance glycoprotein associated with the anticancer effects of berberine, was found to upregulate p-mTOR expression and inhibit cell death in SMMC7721 cells co-treated with rapamycin and berberine. In conclusion, the findings of the present study suggest that the combined use of rapamycin and berberine may improve HCC therapy through synergistically inhibiting the mTOR signaling pathway, which is at least in part, mediated through CD147.

\section{Introduction}

Rapamycin, also termed sirolimus, is a carboxycyclic lactone-lactam macrolide produced by Streptomyces hygroscopicus. It was first identified as an antifungal agent, was subsequently investigated as an immunosuppressant and is clinically used during organ transplantation (1). Rapamycin revealed the mammalian target of rapamycin (mTOR) signaling pathway, which is important for normal cell and cancer cell growth (2). mTOR is found in two different complexes within the cell, mTORC1 and mTORC2; however, only mTORC1 is sensitive to inhibition by rapamycin. Activated mTORC1 regulates protein synthesis through directly phosphorylating 4E-binding protein 1 and p70S6 kinase 1 (p70S6K1), which are translation initiation factors that are important for cap-dependent mRNA translation, thus, activated mTORC1 increases the levels of numerous proteins that are required for cell cycle progression, proliferation, angiogenesis and survival. Several oncogenes and tumor suppressor genes, which activate mTORC1 primarily through the phosphatidylinositol 3-kinase $(\mathrm{PI} 3 \mathrm{~K}) / \mathrm{Akt}$ pathway, are frequently dysregulated in the majority of types of human cancer, including hepatocellular carcinoma (HCC) $(3,4)$. A large number of preclinical and clinical trials have demonstrated that inhibition of the mTOR signaling pathway using rapamycin or rapamycin analogs may be a promising therapeutic strategy for HCC (5-8). Despite the potential use of rapamycin as a chemotherapeutic agent, the immunosuppressive effect simultaneously induced by rapamycin is problematic. Thus, the identification of other classes of anticancer agents, which act synergistically with 
rapamycin are urgently required in order to minimize the immunosuppressive effect.

Berberine, a small molecule derived from Coptidis rhizoma, also termed Huang lian, as well as various other plants, has strong anticancer properties with no significant side effects $(9,10)$. Berberine has been shown to simultaneously induce autophagic cell death and mitochondrial apoptosis in HCC cells (11). In a previous study, we demonstrated that berberine induces cell death in HCC cells through downregulating cluster of differentiation (CD) 147 (12), a transmembrane glycoprotein that is highly expressed in HCC cells and is strongly associated with HCC progression and prognosis (13). Berberine has been reported to inhibit the proliferation and development of HCC cells through inhibiting the mTOR-signaling pathway (11), thus in the present study, it was hypothesized that the combination of rapamycin and berberine may increase the efficacy of chemotherapy for HCC through synergistic suppression of the mTOR signaling pathway. The rapamycin-induced immunosuppressive effect is dose-dependent (1); therefore, the present study investigated the synergistic anticancer effect of different concentrations of rapamycin $(0,10,50$, 100 and $200 \mathrm{nM})$ combined with berberine $(62.5 \mu \mathrm{M})$ on HCC cells in vitro. The possible underlying molecular mechanisms were also investigated.

\section{Materials and methods}

Materials. SMMC7721 and HepG2 human hepatoma cell lines were provided by the Institute of Biochemistry and Cell Biology, Chinese Academy of Sciences (Shanghai, China). RPMI-1640 medium and fetal bovine serum (FBS) were obtained from Gibco-BRL (Grand Island, NY, USA). Berberine and rapamycin were purchased from Sigma-Aldrich (St. Louis, MO, USA). Berberine was dissolved in RPMI-1640 medium at a final concentration of $1 \mathrm{M}$, which was used as a stock solution. Rapamycin was dissolved in dimethyl sulfoxide (DMSO) at a final concentration of $2 \mathrm{mmol} / \mathrm{l}$. 3-[4, 5-Dimethylthiazol-2-yl]-2, 5-diphenyltetrazolium bromide (MTT) was purchased from Sigma-Aldrich. The autophagy inhibitor, 3-methyladenine (3-MA), was obtained from Santa Cruz Biotechnology, Inc. (Santa Cruz, CA, USA). The caspase-3 inhibitor, z-DEVD-fmk (10 mM stock solution) was purchased from BD Biosciences (Franklin Lakes, NJ, USA). The mouse anti-human CD147 and mouse anti-human $\beta$-actin monoclonal antibodies (mAbs) were purchased from BioVision, Inc. (Palo Alto, CA, USA) and the horseradish peroxidase (HRP)-labeled goat anti-mouse immunoglobulin $\mathrm{G}(\mathrm{IgG})$ was purchased from Invitrogen Life Technologies (Carlsbad, CA, USA). The mouse anti-human phosphorylated (p)-mTOR, p70S6K1 and p-p70S6K1 (Thr389) mAbs were purchased from Cell Signaling Technology, Inc. (Beverly, MA, USA). pcDNA3.1 and pcDNA3.1-CD147 were obtained from the Cell Biology Department of The Fourth Military Medical University (Xi'an, China).

Cell culture. Human SMMC7721 and HepG2 cells were cultured in RPMI-1640 supplemented with 10\% FBS and antibiotics (100 U/ml penicillin and $100 \mu \mathrm{g} / \mathrm{ml}$ streptomycin) at $37^{\circ} \mathrm{C}$ in a humidified atmosphere containing $5 \% \mathrm{CO}_{2}$ and
95\% air. To investigate the synergistic effect of berberine- and rapamycin-induced cell death, the SMMC7721 and HepG2 cells were cultured either with or without berberine and rapamycin at $37^{\circ} \mathrm{C}$ for $24 \mathrm{~h}$.

Cell growth and proliferation assay. Cell viability was determined using the MTT quantitative colorimetric assay. Our previous study showed that berberine inhibited HCC cell growth in a dose- and time-dependent manner at a concentration of $62.5 \mu \mathrm{M}$ (12); thus in the present study, berberine was used at a concentration of $62.5 \mu \mathrm{M}$ in combination with rapamycin. In brief, SMMC7721 and HepG2 cells were plated at a density of $5 \times 10^{3}$ cells/well on 96 -well culture plates in RPMI-1640 medium supplemented with 10\% FBS. Following overnight incubation, the cells were treated with various concentrations of rapamycin $(0,10,50,100$ or $200 \mathrm{nM})$ with or without $62.5 \mu \mathrm{M}$ berberine for $24 \mathrm{~h}$. The effect of the combined treatment of berberine with rapamycin was assessed at different time points $(0,12,24,48$ and $72 \mathrm{~h})$. A total of $10 \mu \mathrm{l}$ of $5 \mathrm{mg} / \mathrm{ml} \mathrm{MTT}$ was then added to each well for an additional $4 \mathrm{~h}$ and the resulting formazan crystals were dissolved in $100 \mu \mathrm{l}$ DMSO. The absorbance was read at $570 \mathrm{~nm}$ using an automatic microplate reader (Immuno Mini NJ-2300; Intermed Inc., Tokyo, Japan).

Analysis of cell death using transmission electron microscopy (TEM). TEM analysis was performed as described previously (14). In brief, following culture with $200 \mathrm{nM}$ rapamycin in the absence or presence of $62.5 \mu \mathrm{M}$ berberine for $24 \mathrm{~h}$, SMMC7721 and HepG2 cells were fixed using 3\% glutaraldehyde in 0.2 M phosphate-buffered saline (PBS; pH 7.3) for $4 \mathrm{~h}$ at $4^{\circ} \mathrm{C}$. The cells were then post-fixed with $1 \%$ osmium tetroxide and $0.5 \%$ tannic acid for $1 \mathrm{~h}$ at $4^{\circ} \mathrm{C}$, and washed three times using 0.1 M PBS ( $\mathrm{pH}$ 7.3). Cells were dehydrated and embedded in EPON 812 (Electron Microscopy Sciences, Fort Washington, PA, USA). Sections were then counterstained with uranyl acetate and lead citrate, and analyzed using a JEM-2000EX transmission electron microscope (Jeol Ltd., Tokyo, Japan).

Trypan blue exclusion assay. Cell death was analyzed using a Trypan blue exclusion assay as described previously (15). In brief, SMMC7721 cells were cultured for $24 \mathrm{~h}$ with $62.5 \mu \mathrm{M}$ berberine and $50 \mathrm{nM}$ rapamycin in the absence or presence of the autophagy inhibitor 3-MA (10 $\mathrm{mM})$ and/or the caspase-3 inhibitor z-DEVD-fmk $(100 \mu \mathrm{M})$. Adherent and non-adherent cells were harvested, washed three times with PBS and resuspended in $100 \mu \mathrm{l}$ PBS. Subsequent to mixing with $100 \mu \mathrm{l}$ of $0.8 \%$ Trypan blue, cells were counted using a hemocytometer (Yancheng Hengtai Glass Instrument, Yancheng, China). The number of dead cells with disrupted membranes (blue cells) per 200 cells was counted in three replicates. Cell death was expressed as the mean percentage of blue cells/total cells.

Western blot analysis. Western blot analysis was performed as described previously (16). In brief, cells were lysed on ice for $30 \mathrm{~min}$ in radioimmunoprecipitation assay buffer (Beyotime Biotechnology Inc., Nantong, China) containing $100 \mu \mathrm{M}$ phenylmethylsulfonyl fluoride. Following centrifugation at $12,000 \mathrm{x} \mathrm{g}$ for $15 \mathrm{~min}$, the supernatant was harvested 
A

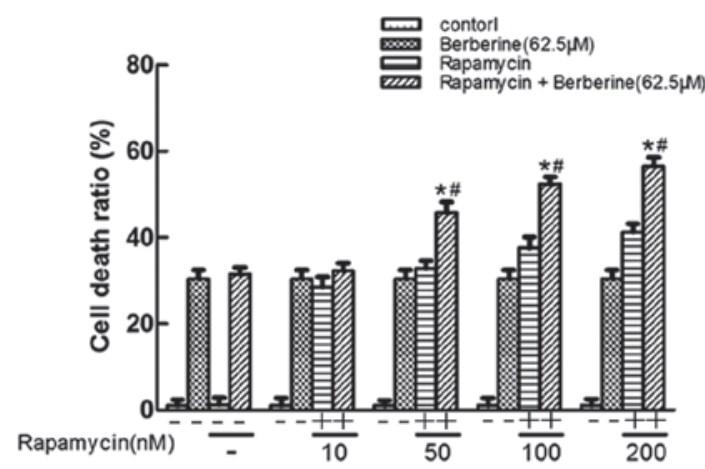

C

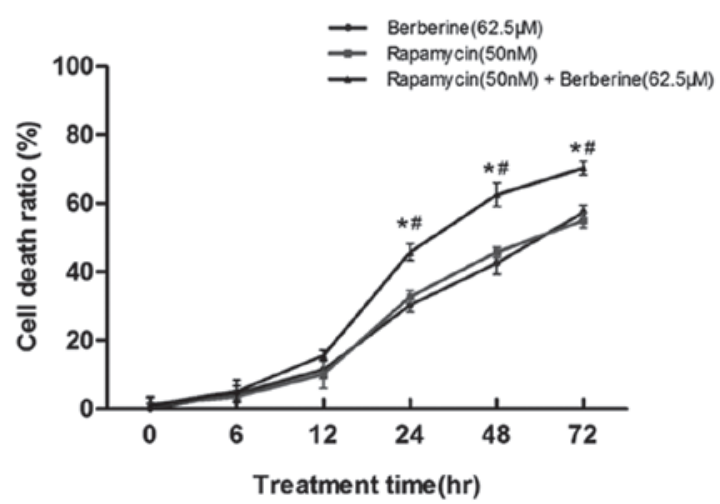

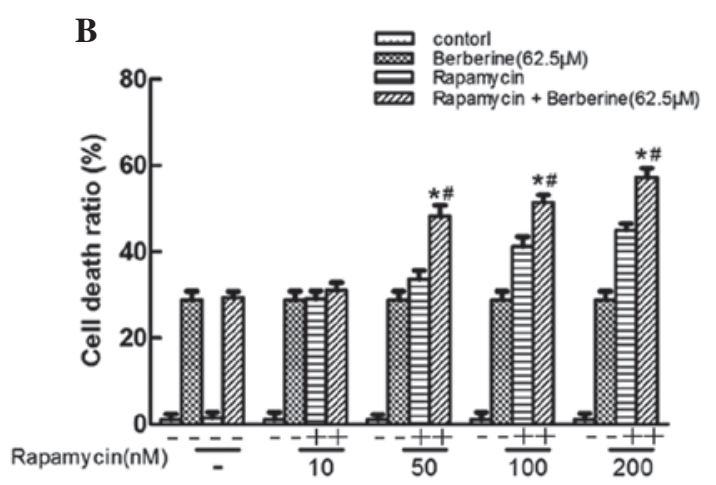

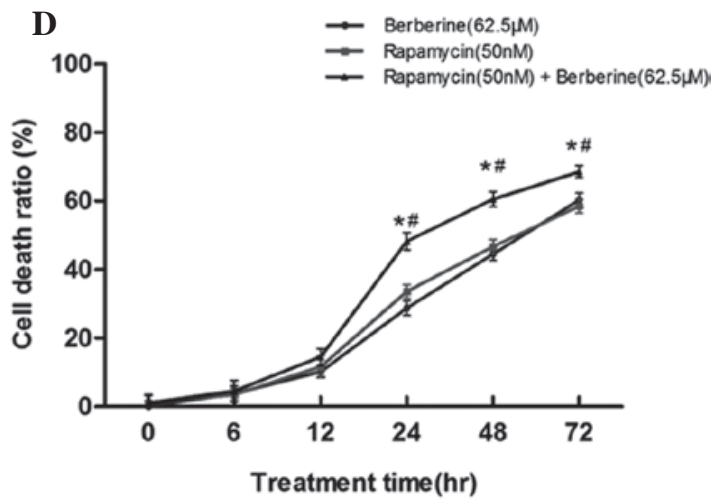

Figure 1. Combined effect of berberine and rapamycin on the viability of (A and C) SMMC7721 cells and (B and D) HepG2 cells. (A and B) Combined berberine and rapamycin treatment decreased the viability of SMMC7721 and HepG2 cells in a dose-dependent manner. Cells were treated with various concentrations of rapamycin and $62.5 \mu \mathrm{M}$ berberine for $24 \mathrm{~h}$. Cell viability was then determined using MTT assay. (C and D) Combined berberine and rapamycin treatment decreased the viability of SMMC7721 and HepG2 cells in a time-dependent manner. Cells were treated with berberine and rapamycin for the indicated durations. Cell viability was then determined using MTT assay. ${ }^{*} \mathrm{P}<0.05$ vs. berberine-treated cells, ${ }^{*} \mathrm{P}<0.05$ vs. rapamycin-treated cells.

as the total cellular protein extract and was stored at $-70^{\circ} \mathrm{C}$. The protein concentration was determined using a bicinchoninic acid kit (Pierce Biotechnology, Inc., Rockford, IL, USA). Equal quantities of total protein were separated using $10 \%$ SDS-PAGE, then transferred to polyvinylidene fluoride membranes (Millipore Corporation, Billerica, MA, USA). Membranes were blocked with 5\% non-fat dry milk in PBS containing $0.05 \%$ Tween-20 and incubated with antibodies against p70S6K1, p-p70S6K1, CD147, p-mTOR and $\beta$-actin. Membranes were washed three times in PBS, followed by incubation with the appropriate HRP-linked IgG.

Overexpression of CD147 in SMMC7721 cells. We previously reported that $\mathrm{CD} 147$ has an important role in berberine-induced cell death (12). In order to investigate whether CD147 also participates in the regulation of the mTOR pathway, SMMC7721 cells were transfected with pcDNA3.1-CD147 or pcDNA3.1 (Invitrogen Life Technologies, Carlsbad, CA, USA) for $24 \mathrm{~h}$ and then cultured with rapamycin and berberine for $24 \mathrm{~h}$. mTOR expression was assessed using western blot analysis.

Statistical analysis. Statistical analyses were performed using SPSS 13.0 statistical software (SPSS, Inc., Chicago, IL, USA). Data are presented as the mean \pm standard deviation. Statistical significance was determined using Student's t-test and analysis of variance. $\mathrm{P}<0.05$ was considered to indicate a statistically significant difference.

\section{Results}

Berberine enhances rapamycin-mediated cell death in vitro. To determine cell viability following combined treatment with berberine and different concentrations of rapamycin in human SMMC7721 and HepG2 cells, an MTT assay was performed. Initially, the separate effects of rapamycin and berberine were investigated on the viability of SMMC7721 and HepG2 cells. In our previous study (12), $62.5 \mu \mathrm{M}$ berberine was combined with rapamycin. In the present study, the HCC cells were treated with various doses of rapamycin for $24 \mathrm{~h}$ and cell viability was subsequently measured. As shown in Fig. 1A and B, the cell death ratio was significantly increased by rapamycin in a dose-dependent manner. Furthermore, berberine was found to significantly enhance cell cytotoxicity when combined with $50 \mathrm{nM}$ rapamycin. Similar anticancer effects were observed in the cells treated with $62.5 \mu \mathrm{M}$ berberine and $50 \mathrm{nM}$ rapamycin and those treated with 100-200 nM rapamycin, indicating that berberine synergistically enhanced rapamycin-mediated cell death in vitro. Thus, $62.5 \mu \mathrm{M}$ berberine and $50 \mathrm{nM}$ rapamycin was used in order to analyze the combined effect of berberine and rapamycin in the HCC cells. The present study also investigated whether the combined effect of rapamycin and berberine on cell viability was time-dependent. Fig. $1 \mathrm{C}$ and D show that combined treatment with berberine and rapamycin induced cell death in a time-dependent manner in SMMC7721 and HepG2 cells. 
A

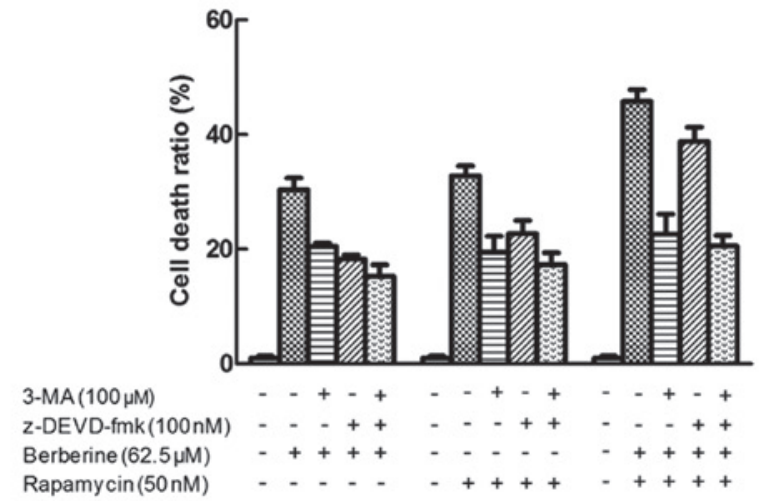

B

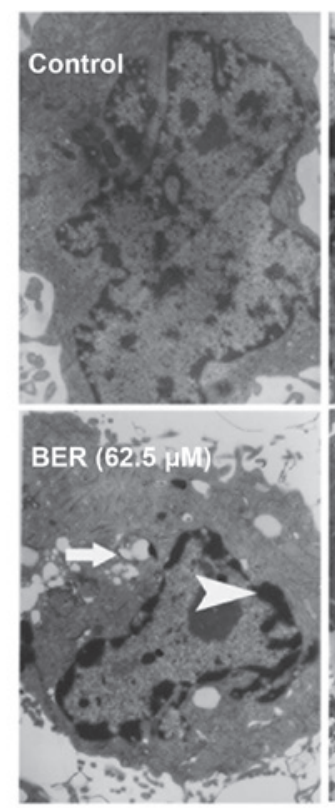

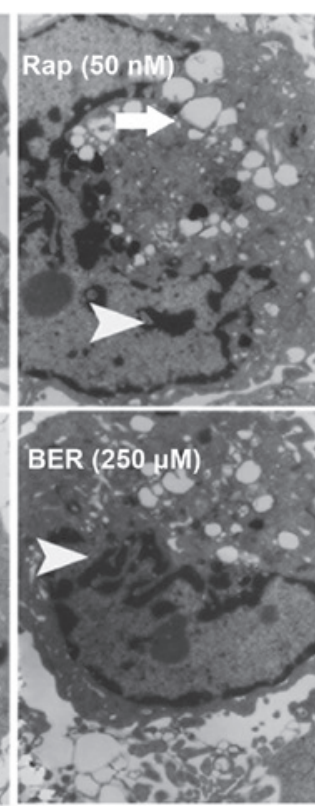

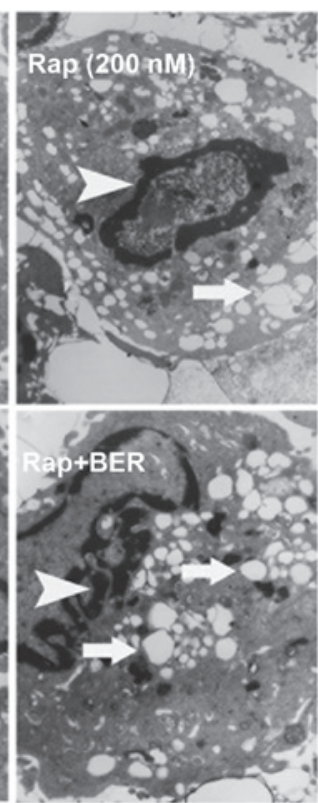

Figure 2. Cell death induced through combined berberine and rapamycin treatment in SMMC7721 cells. (A) SMMC7721 cells were treated with $62.5 \mu$ M berberine or $50 \mathrm{nM}$ rapamycin alone or co-treated with berberine and rapamycin in the absence or presence of 3-MA and/or z-DEVD-fmk. 3-MA and z-DEVD-fmk inhibited cell death. The mean percentage of cell death from triplicate samples is shown for the different treatment groups. Data are presented as the mean \pm standard deviation. (B) SMMC7721 cells were treated with single doses of berberine (62.5 or $250 \mu \mathrm{M})$ or rapamycin (50 or $200 \mathrm{nM})$ or co-treated with $62.5 \mu \mathrm{M}$ berberine and $50 \mathrm{nM}$ rapamycin, prior to harvesting and analysis using transmission electron microscopy. The white arrows indicate autophagic vesicles and the white arrowheads indicate apoptosis. 3-MA, 3-methyladenine; Ber, berberine; Rap, rapamycin.

\section{p-p70S6K1}

p70S6K1

\section{$\beta$-actin}

Berberine
Rapamycin

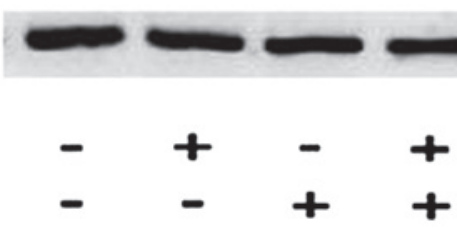

Figure 3. Combined treatment with berberine and rapamycin decreases p-p70sk61 expression in SMMC7721 cells. SMMC7721 cells were treated with rapamycin in the absence or presence of berberine for $12 \mathrm{~h}$, then p70S6K1 and p-p70S6K1 expression was detected using western blot analysis. Treatment with rapamycin or berberine alone significantly inhibited the protein expression of p-p70S6K1, but not p70S6K1. Moreover, a significantly higher reduction in $\mathrm{p}-\mathrm{p} 70 \mathrm{~S} 6 \mathrm{~K} 1$ protein expression was observed following co-treatment with berberine and rapamycin. p-, phosphorylated; p70S6K1, p70S6 kinase 1.

Autophagy is induced by combined berberine and rapamycin treatment in HCC cells. To determine the specific type of cell death induced by berberine and rapamycin, the effect of the combined treatment was assessed in the SMMC7721 cells using a Trypan blue exclusion assay. 3-MA, a common autophagy inhibitor, was used to inhibit autophagy and prevent autophagic cell death. 3-MA does not inhibit cell death induced by other mechanisms. z-DEVD-fmk, an apoptosis inhibitor, inhibits apoptosis, but no other types of cell death. In the present study, berberine-induced and rapamycin-induced cell death were observed to involve autophagy and apoptosis (Fig. 2A). Furthermore, the combined use of 3-MA and z-DEVD-fmk did not completely inhibit cell death, indicating that cell death may also be induced by other mechanisms, including necrosis. However, upon combined treatment with berberine and rapamycin, a significantly stronger inhibition of 3-MA- rather than z-DEVD-fmk-induced cell death was observed, suggesting that berberine may enhance rapamycin-induced cell death primarily through autophagy.

To further analyze the synergistic effect of berberine and rapamycin on autophagy, the level of autophagy was assessed in SMMC7721 cells using TEM. Autophagic vacuoles were observed in the rapamycin-treated SMMC7721 cells (Fig. 2B). However, a significantly higher level of autophagy was found in the SMMC7721 cells co-treated with berberine and rapamycin compared with those treated with rapamycin alone. As shown in Fig. 2B, treatment with $50 \mathrm{nM}$ rapamycin induced significant cell autophagy when combined with $62.5 \mu \mathrm{M}$ berberine compared with treatment with $200 \mathrm{nM}$ rapamycin, indicating that the combined use of berberine and rapamycin had a synergistic effect on cell autophagy.

Combined berberine and rapamycin-induced cell death is associated with inhibition of mTOR-p70S6K1 activity. p70S6K1 is a downstream target of mTOR. To determine whether the phosphorylation of p70S6K1 is involved in berberine and rapamycin-induced cell death in HCC cells, SMMC7721 cells were treated with rapamycin in the absence or presence of berberine for $12 \mathrm{~h}$ and the phosphorylation of p70S6K1 at Ser421/Thr424 was detected using western blot analysis. As shown in Fig. 3, rapamycin and berberine alone were not 
A

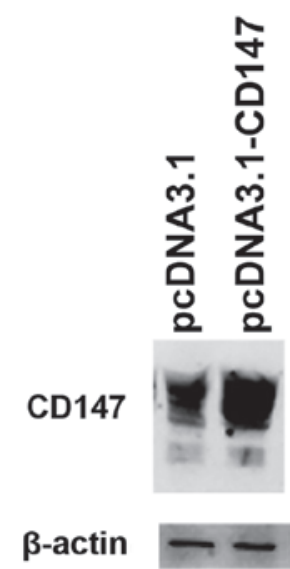

C

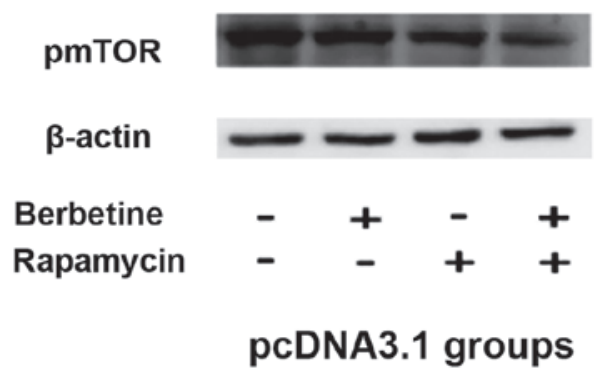

B

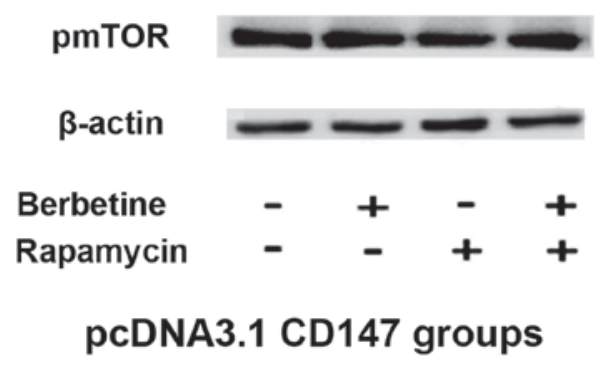

D

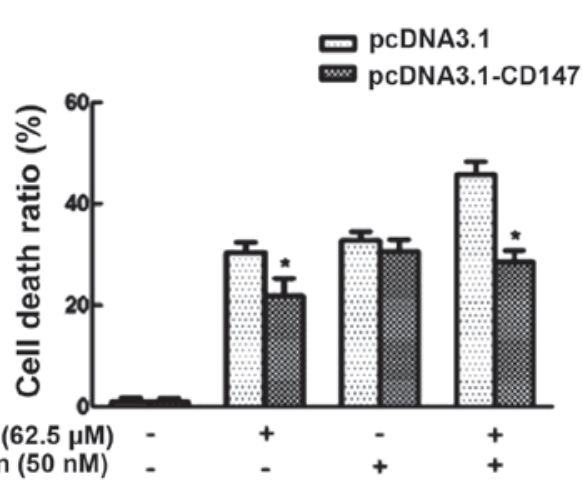

Figure 4. Overexpression of CD147 in SMMC7721 cells decreases cell death and inhibits mTOR downregulation upon co-treatment with berberine and rapamycin. (A) Overexpression of CD147 induced by transfecting SMMC7721 cells with pcDNA3.1-CD147. (B) mTOR expression was detected following SMMC7721 cell transfection with pcDNA3.1-CD147. No significant reduction in mTOR expression was observed in the SMMC7721 cells transfected with pcDNA3.1-CD147. (C) A significant decrease in mTOR expression was observed in the pcDNA3.1-transfected SMMC7721 cells co-treated with berberine and rapamycin compared with those treated with berberine or rapamycin alone. (D) SMMC7721 cells transfected with pcDNA3.1-CD147 exhibited significantly reduced cell death compared with those transfected with pcDNA3.1 upon co-treatment with rapamycin and berberine rather than berberine or rapamycin alone. $\mathrm{CD}$, cluster of differentiation; pmTOR, phosphorylated mammalian target of rapamycin.

found to significantly inhibit the phosphorylation of p70S6K1. Moreover, a significantly higher reduction in p70S6K1 phosphorylation was observed following berberine addition. These results suggest that the inhibition of p70S6K1 activation was associated with berberine and rapamycin-induced cell death.

Inhibitory effect of berberine and rapamycin on p-mTOR is associated with CD147. Our previous studies have suggested that berberine induces cell death in HCC cells through the downregulation of CD147. In order to investigate whether CD147 was involved in the cell death induced by combined berberine and rapamycin treatment, CD147 was overexpressed in SMMC7721 cells through transfection with pcDNA3.1-CD147. p-mTOR expression was then detected using western blot analysis and cell death was assessed using the Trypan blue exclusion assay. As indicated in Fig. 4A, following transfection with pcDNA3.1-CD147, CD147 was found to be significantly overexpressed in SMMC7721 cells. Furthermore, a more significant decrease in p-mTOR expression was observed following combined treatment with berberine and rapamycin compared with treatment with berberine or rapamycin alone (Fig. 4C). However, no significant reduction in p-mTOR expression was observed in SMMC7721 cells transfected with pcDNA3.1-CD147 (Fig. 4B). SMMC7721 cells transfected with pcDNA3.1-CD147 exhibited significantly lower levels of cell death, as compared with those transfected with pcDNA3.1, upon berberine treatment alone or co-treatment of berberine with rapamycin (Fig. 4D). These results suggest that overexpression of CD147 inhibits berberine and rapamycin-induced cell death.

\section{Discussion}

While the inhibition of mTOR represents an attractive anticancer target and clinical trials of rapamycin and its analogs have shown the importance of mTOR inhibition as a novel treatment strategy for malignancies, rapamycin-induced immunosuppression is a problem preventing its clinical application. Rapamycin-induced immunosuppression is dose-dependent and analyses of transplant patients who were administered rapamycin have revealed that through careful therapeutic drug monitoring, it is possible to maximize the benefits and minimize the hazards of chronic immunosuppression using a rapamycin-based regimen (1). Thus, rapamycin-induced immunosuppression may be overcome through combining lower concentrations of rapamycin with other anticancer agents. Berberine has been demonstrated to have strong anticancer properties in HCC cells, which involve inhibition of 
the mTOR-signaling pathway through suppressing the activity of Akt (11). Therefore, in the present study, the anticancer effect of berberine combined with various concentrations of rapamycin was investigated in HCC cells in vitro. Potent synergistic cytotoxic effects were observed upon co-treatment with berberine and rapamycin in SMMC7721 and HepG2 cells. Furthermore, berberine was found to maintain the cytotoxic effects of rapamycin on the HCC cells with a lower concentration of rapamycin, suggesting that berberine may minimize rapamycin-induced immunosuppression by allowing a lower dose of rapamycin to be used. Similar synergistic anticancer results have been reported in SH-SY5Y human neuroblastoma cells, where a low dose combination of berberine and $\mathrm{As}_{2} \mathrm{O}_{3}$, an anticancer agent that induces various side effects at high concentrations, was found to markedly decrease cell viability compared with treatment with a low dose of berberine or $\mathrm{As}_{2} \mathrm{O}_{3}$ alone (17). Berberine is a small molecule, which is easily isolated from a large number of traditional medicinal plants and has strong anticancer properties without significant side effects $(9,10)$. Thus, the findings of the present study suggest that the combined use of berberine with low doses of rapamycin may be a novel and promising chemotherapeutic strategy for HCC treatment, with minimal rapamycin-induced immunosuppressive effects. However, further investigations are required using in vivo $\mathrm{HCC}$ models.

The mode of cell death induced by combined berberine and rapamycin treatment in HCC cells was also investigated. The PI3K/Akt/mTOR pathway has been characterized as a key negative regulator of apoptosis and autophagy in a number of types of cancer cell and has been proposed to control cellular processes that contribute to the initiation and maintenance of cancer. Previous studies have demonstrated that berberine simultaneously induces autophagic cell death and apoptosis in HCC cells $(11,12)$. In the present study, apoptosis and autophagy were simultaneously observed in HCC cells treated with berberine combined with rapamycin. In addition, autophagic cell death was observed to be more prominent than apoptosis in the cell death induced by these two agents.

Notably, the incubation of HCC cells with 3-MA combined with z-DEVD-fmk was not found to completely prevent cell death induced by combined berberine and rapamycin treatment. This finding suggests that besides autophagic cell death and apoptosis, other types of cell death, including necrosis may be induced in the HCC cells upon co-treatment with berberine and rapamycin. This was consistent with the findings of our previous study (12) and is in agreement with a previous report showing that berberine induces cell lysis/necrosis in B16 murine melanoma cells (18).

To determine the possible signaling molecules responsible for the synergistic cell killing effect induced by berberine and rapamycin, the level of p-p70S6K1 (the downstream target of mTOR) was investigated. p-p70S6K1 expression was found to be significantly inhibited by treatment with berberine and rapamycin alone. Moreover, combined treatment with berberine and rapamycin induced a significant reduction in the level of p-p70S6K1 in the HCC cells compared with that in those treated with rapamycin or berberine alone. This suggests that p70S6K1, as well as mTOR, may be the target molecules involved in the synergistic cell killing effect of combined berberine and rapamycin treatment, and that the efficacy of this combination may have been induced through further suppression of mTOR signaling. This was further supported by the findings of the present study, that in SMMC7721 cells transfected with pcDNA3.1, berberine and rapamycin alone were capable of inhibiting p-mTOR expression, while the combination of berberine and rapamycin induced a lower level of p-mTOR than berberine or rapamycin alone. Similar synergistic mechanisms have been reported in HCC cells co-treated with the rapamycin analog RAD001 and the Akt inhibitor MK-2206 (19), as well as in HCC cells co-treated with rapamycin and bortezomib, a proteasome inhibitor with antitumor activity, including the capacity to downregulate p-Akt (20). However, in the present study, whether there were other mechanisms involved in the synergistic anticancer effect of combined berberine and rapamycin treatment, other than the synergistic suppression of mTOR signaling, requires further investigation.

In our previous study, berberine was found to induce cell death in SMMC7721 cells through downregulating CD147 (12). Furthermore, CD147 has been reported to inhibit starvation-induced autophagic cell death in SMMC7721 cells through increasing the activity of the PI3K/Akt pathway (16). To the best of our knowledge, at present, no studies have directly focused on the interrelation between CD147 and mTOR signaling. In order to investigate whether CD147 was implicated in the cell death mediated by mTOR signaling in the HCC cells co-treated with berberine and rapamycin, CD147 was overexpressed in SMMC7721 cells and its effect on cell death was observed. Overexpression of CD147 was found to significantly inhibit the downregulation of $\mathrm{p}$-mTOR expression and to decrease cell death in cells co-treated with berberine and rapamycin. Based on these findings and our previous studies $(12,16)$, it was hypothesized that CD147 may have a regulatory effect on the activity of the PI3K/Akt/mTOR signaling pathway, and that the cell death induced by combined berberine and rapamycin treatment may, at least in part, be due to berberine-induced CD147 downregulation. However, the effect of rapamycin on CD147 expression in $\mathrm{HCC}$ cells requires further investigation.

In conclusion, the results of the present study indicate that berberine sensitizes rapamycin-mediated HCC cell death, at least in part, through synergistically inhibiting mTOR signaling through the downregulation of CD147. These findings suggest the possibility of developing a novel regimen capable of improving HCC therapy and minimizing the immunosuppression associated with rapamycin through decreasing its dose.

\section{Acknowledgements}

The present study was supported by grants from the National Natural Science Foundation of China (no. 81101744), the Natural Science Foundation of Xi'an Medical University (no. 11FZ09 and 11FZ14) and the Scientific Research Program, funded by Shaanxi Provincial Health Department (nos. 2014D21 and 2014D24).

\section{References}

1. Saunders RN, Metcalfe MS and Nicholson ML: Rapamycin in transplantation: a review of the evidence. Kidney Int 59: 3-16, 2001. 
2. GibbonsJJ,AbrahamRT and YuK:Mammalian target of rapamycin: discovery of rapamycin reveals a signaling pathway important for normal and cancer cell growth. Semin Oncol 36 (Suppl 3): S3-S17, 2009.

3. Zhou L, Huang Y, Li J and Wang Z: The mTOR pathway is associated with the poor prognosis of human hepatocellular carcinoma. Med Oncol 27: 255-261, 2010.

4. Owonikoko TK and Khuri FR: Targeting the PI3K/AKT/mTOR pathway: biomarkers of success and tribulation. Am Soc Clin Oncol Educ Book 2013: 395-401, 2013.

5. Finn RS, Poon RT, Yau T, et al: Phase I study investigating everolimus combined with sorafenib in patients with advanced hepatocellular carcinoma. J Hepatol 59: 1271-1277, 2013.

6. Fasolo A and Sessa C: Targeting mTOR pathways in human malignancies. Curr Pharm Des 18: 2766-2777, 2012

7. Zhou Q, Lui VW and Yeo W: Targeting the PI3K/Akt/mTOR pathway in hepatocellular carcinoma. Future Oncol 7: 1149-1167, 2011.

8. Kudo M: mTOR inhibitor for the treatment of hepatocellular carcinoma. Dig Dis 29: 310-315, 2011.

9. Sun Y, Xun K, Wang Y and Chen X: A systematic review of the anticancer properties of berberine, a natural product from Chinese herbs. Anticancer Drugs 20: 757-769, 2009.

10. Tang J, Feng Y, Tsao S, Wang N, et al: Berberine and Coptidis rhizoma as novel antineoplastic agents: a review of traditional use and biomedical investigations. J Ethnopharmacol 126: 5-17, 2009.

11. Wang N, Feng Y, Zhu M, et al: Berberine induces autophagic cell death and mitochondrial apoptosis in liver cancer cells: the cellular mechanism. J Cell Biochem 111: 1426-1436, 2010.

12. Hou Q, Tang X, Liu H, et al: Berberine induces cell death in human hepatoma cells in vitro by downregulating CD147. Cancer Sci 102: 1287-1292, 2011
13. Tang X, Guo N, Xu L, et al: CD147/EMMPRIN: an effective therapeutic target for hepatocellular carcinoma. J Drug Target: Aug 29,2012 (Epub ahead of print).

14. Espert L, Denizot M, Grimaldi M, et al: Autophagy is involved in T cell death after binding of HIV-1 envelope proteins to CXCR4 J Clin Invest 116: 2161-2172, 2006

15. Valentim L, Laurence KM, Townsend PA, et al: Urocortin inhibits Beclin1-mediated autophagic cell death in cardiac myocytes exposed to ischaemia/reperfusion injury. J Mol Cell Cardiol 40: 846-852, 2006.

16. Gou X, Ru Q, Zhang H, et al: HAb18G/CD147 inhibits starvation-induced autophagy in human hepatoma cell SMMC7721 with an involvement of Beclin 1 down-regulation. Cancer Sci 100: 837-843, 2009.

17. Kim DW, Ahan SH and Kim TY: Enhancement of arsenic trioxide $(\mathrm{As}(2) \mathrm{O}(3))$-mediated apoptosis using berberine in human neuroblastoma SH-SY5Y cells. J Korean Neurosurg Soc 42: 392-399, 2007.

18. Letasiová S, Jantová S, Cipák L and Múcková $\mathrm{M}$ Berberine-antiproliferative activity in vitro and induction of apoptosis/necrosis of the U937 and B16 cells. Cancer Lett 239: 254-262, 2006.

19. Grabinski N, Ewald F, Hofmann BT, et al: Combined targeting of AKT and mTOR synergistically inhibits proliferation of hepatocellular carcinoma cells. Mol Cancer 11: 85, 2012.

20. Wu C, Miao X, Huang L, et al: Genome-wide association study identifies five loci associated with susceptibility to pancreatic cancer in Chinese populations. Nat Genet 44: 62-66, 2011. 